\title{
Tumescent Anesthesia in Skin- and Nipple-sparing Mastectomy: Results of a Prospective Clinical Study
}

\author{
MARCO GIPPONI ${ }^{1}$, ILARIA BALDELLI ${ }^{2}$, GIULIA ATZORI ${ }^{1}$, PIERO FREGATTI ${ }^{1}$, FEDERICA MURELLI ${ }^{1}$, \\ MARIANNA PESCE ${ }^{2}$, CECILIA MARGARINO $^{1}$, FRANCESCA DEPAOLI $^{1}$ and DANIELE FRIEDMAN ${ }^{1}$ \\ ${ }^{1}$ Breast Surgery and ${ }^{2}$ Plastic Surgery, Breast Unit, University of Genoa, School of Medicine, \\ San Martino Hospital and National Cancer Institute, Genoa, Italy
}

\begin{abstract}
Background: The tumescent mastectomy technique has been used to facilitate dissection of subcutaneous tissue and mammary gland in order to reduce intraoperative bleeding and speed the operation. Patients and Methods: A prospective clinical study was performed on 30 female patients undergoing immediate breast reconstructions after skin-sparing mastectomy (SSM) or nipple-sparing mastectomy (NSM) in order to assess early postoperative complications, pain, and final esthetic outcome of skin flaps related to tumescent anesthesia (TA). Results: TA significantly speeded-up the operative procedure (131 \pm 49.99 vs. $180.5 \pm 67.15$ min; $p=0.03$ ) achieving less skin damage compared to patients who did not have TA ( $p=0.045)$; moreover, no significant difference occurred with regard to the length of in-hospital stay and overall lymphatic drainage. Conclusion: The length of the operation as well as the final cosmetic outcome of skin flaps was significantly improved due to TA, with no appreciable side-effects.
\end{abstract}

Skin-sparing mastectomy (SSM) and nipple-sparing mastectomy (NSM) have greatly improved the cosmetic outcome in patients with breast cancer without impairing the locoregional control of the disease. Tumescent anesthesia (TA) has been used to facilitate dissection of and between subcutaneous tissue and mammary gland, reducing intraoperative bleeding and operative time. Its application was initially proposed in liposuction, and it consists of injecting a very dilute solution of local anesthetic combined with epinephrine and sodium bicarbonate into the tissues until they become firm and tense (tumescent) (1). As originally described by Klein, the slow infiltration of a local

Correspondence to: Marco Gipponi, MD, Breast Unit - IRCCS San Martino-IST Genoa, L.go R. Benzi, 10, 16132 Genoa, Italy. E-mail: marco.gipponi@hsanmartino.it

Key Words: Breast cancer, breast reconstruction, tumescent anesthesia. anesthetic solution of lidocaine and epinephrine minimizes the rate of systemic absorption and reduces the potential for toxicity; moreover, the dilution of lidocaine $(0.05 \%$ of $0.1 \%)$ and epinephrine $(1: 1,000,000)$ further delays absorption and reduces the magnitude of peak plasma lidocaine concentration (2). The peculiar features of TA allow: i) hydrostatic dissection with rapid flap development using a scalpel or electrocauterizer with minimal blood loss, thanks also to the epinephrine-induced vasoconstriction; ii) local analgesia due to the anesthetic solution; and iii) reduced thermo-dispersion of the electrocauterizer with fewer skin and subcutaneous ischemic lesions.

However, controversy especially exists regarding the risk of skin-flap necrosis induced by TA itself when mastectomy is associated with breast reconstruction. Although most previous studies are retrospective, they do not relate to a uniform surgical technique and the pharmacological composition of the injected solution was frequently different (3-10).

This prospective clinical study included a surgeondependent auto-randomization of patients with the aim of evaluating the effect of TA without epinephrine previous to the scheduled operation performed according to a standardized surgical technique.

\section{Patients and Methods}

From February to May 2015, 30 patients underwent immediate alloplastic breast reconstruction after SSM or NSM at the Breast Unit of San Martino Hospital and National Cancer Institute in Genoa. Patients were divided into two groups according to their surgeon's preference regarding the use or not of TA. Surgeon A used the standard dry surgical technique, while surgeon B performed a modified tumescent mastectomy under general anesthesia. The breast reconstruction was performed by the same plastic surgeon who was not aware of the surgeon-related selection of patients. Patients older than 75 years old, who had undergone neoadjuvant chemotherapy, pregnant women or patients who were not available for/compliant with outpatient follow-up were excluded from this study. Specific informed consent was signed by all the patients. The study was conducted in accordance with Good Clinical Practice and the Declaration of Helsinki. 
Preoperative data included the assessment of co-morbid conditions such as: hypertension, coronary artery disease; chronic obstructive pulmonary disease; congestive heart failure; cerebral vascular disease; diabetes mellitus, and smoking habits. Based on the patient's body mass index (BMI), four categories of patients were defined: underweight $\left(<18.5 \mathrm{~kg} / \mathrm{m}^{2}\right)$; normal weight $\left(18.5-24.9 \mathrm{~kg} / \mathrm{m}^{2}\right)$; overweight $\left(25.0-29.9 \mathrm{~kg} / \mathrm{m}^{2}\right)$, and obese $\left(>30 \mathrm{~kg} / \mathrm{m}^{2}\right)$. Moreover breast size was indirectly assessed by means of the bra size.

Peri- and postoperative data included: operative time; hospitalization; postoperative pain that was assessed by both daily visual analog scale (VAS) self-assessment and the overall number of tablets of non-steroidal anti-inflammatory drugs (NSAIDs) that were given to the patient; overall lymphatic drainage, and final surgical outcome, especially in regard to the occurrence of skin necrosis (superficial epidermolysis or major tissue necrosis), hematoma, wound infection, or otherwise delayed wound healing. All data were collected by the same physician. Statistical analysis included the mean, standard deviation, and mean standard error for numerical variables; continuous variables were analyzed with a two-tailed Student's $t$-test and binomial variables with Chisquare analysis, and a $p$-value of less than 0.05 was considered statistically significant.

Modified tumescent mastectomy technique. The injected solution was always composed of ropivacaine $150 \mathrm{mg}(7.5 \mathrm{mg} / \mathrm{ml}$; two vials in $250 \mathrm{cc}$ of saline solution). For patients of group B, the solution was injected into the subcutaneous tissue by means of a spinal needle (22-gauge; $30 \mathrm{~mm}$ ) to establish a bloodless plane for surgical dissection. The needle was repeatedly introduced in a radial fashion after performing the skin incision in order to achieve a complete hydro-dissection all around the surgical incision. Thereafter electrocautery was used to dissect out the mastectomy flaps and, as soon as mastectomy was accomplished, all patients underwent immediate breast reconstruction with tissue expander placement

\section{Results}

A total of 30 breast reconstructions with a tissue expander after SSM or NSM were performed in 30 patients after oncological mastectomy. The clinical features of patients and surgical procedures are listed in Table I. Group A included 15 patients who underwent immediate alloplastic breast reconstruction subsequent to standard dry mastectomy performed by Surgeon A. Group B included 15 patients who underwent immediate alloplastic breast reconstruction after modified tumescent mastectomy had been performed by Surgeon B. Overall, axillary lymph-node dissection was associated with 11 procedures.

By comparing groups for clinical characteristics, a statistically significant difference was found for patient age: Group A patients were younger than those in group B (48.26 vs. 53.37 years, respectively; $p=0.047)$. No statistically significant difference was found for patient BMI, smoking habits, diabetes, prior radiotherapy, and co-morbidity factors. Operative time was significantly shorter in group B patients $(p=0.03)$; by comparing groups for immediate surgical outcome, group B patients suffered significantly less skin
Table I. Clinical features and postoperative assessment in patients undergoing standard dry surgical technique (group A) or modified tumescent mastectomy (group B).

\begin{tabular}{lccc}
\hline Parameter & $\begin{array}{c}\text { Group A } \\
(\mathrm{N}=15)\end{array}$ & $\begin{array}{c}\text { Group B } \\
(\mathrm{N}=15)\end{array}$ & $p$-Value \\
\hline Median age (years) & 48.26 & 53.37 & 0.047 \\
Median BMI (kg/m²) & 20.38 & 23.49 & \\
Bra size & 2.3 & 2.9 & 0.07 \\
Smokers, $\mathrm{n}$ & $5(33 \%)$ & $6(40 \%)$ & 0.70 \\
Type of mastectomy, n & & & \\
$\quad$ SSM & 10 & 9 & \\
$\quad$ NSM & 5 & 6 & \\
Additional procedures, $\mathrm{n}$ & & & \\
$\quad$ SLNB alone & 10 & 9 & 0.87 \\
$\quad$ SLNB and AD & 5 & 6 & \\
Operative time (min) & $180.5 \pm 67.15$ & $131 \pm 49.99$ & 0.03 \\
Hospitalization (days) & $5.1 \pm 0.96$ & $5.5 \pm 1.40$ & 0.45 \\
Skin flaps, n & & & \\
$\quad$ Normal & 8 & 13 & 0.045 \\
Superficial necrosis & 7 & 2 & \\
$\quad$ Deep necrosis & 0 & 0 & \\
Postoperative pain (VAS) & $8.5 \pm 2.38$ & $6.7 \pm 2.01$ & 0.45 \\
NSAID use, no. of tablets & $10.5 \pm 3.05$ & $10 \pm 3.53$ & 0.91 \\
Lymphatic drainage (ml) & $374 \pm 177.87$ & $351 \pm 184.37$ & 0.73 \\
\hline
\end{tabular}

BMI, Body mass index; SSM, skin-sparing mastectomy; NSM, nipplesparing mastectomy; SLNB, sentinel lymph-node biopsy; AD, axillary dissection; VAS, Visual Analog Scale; NSAID: non-steroidal antiinflammatory drug. Data are the mean \pm SD or number of patients unless stated otherwise.

damage (superficial epidermolysis or major tissue necrosis) with an improved final cosmetic outcome as compared to group A patients $(p=0.045)$. No significant difference was found for the remaining peri- and postoperative data (hospitalization, postoperative pain, overall lymphatic drainage, hematoma, wound infection, or otherwise delayed wound healing).

\section{Discussion}

TA has been effectively used in breast surgery as well as in other surgical procedures (3-16). As a matter of fact, the injection of tumescent solution was demonstrated to facilitate the creation of a hydro-dissection plane, lead to epinephrineinduced vasoconstriction, reduce the use of electrocautery, and to reduce postoperative pain due to the local anesthetic effect $(17,18)$.

Regarding the use of electrocautery alone, it reduces the intraoperative blood loss per se, but increases the operative time of mastectomy compared to knife or scissor dissection, and may also lead to wider skin necrosis due to its direct thermal damage; conversely, TA may reduce thermodispersion of electrocauterization with fewer skin and 
subcutaneous ischemic lesions, thus achieving not only shorter time of operation but also less incidence and volume of seroma $(19,20)$.

However, the compressive effect on subcutaneous tissues and the vasoconstrictor effect of epinephrine, especially in combination with higher initial tissue expander volume for immediate reconstruction, could potentially contribute to skin-flap ischemia (8). The use of epinephrine may also result in delayed hematoma because of transient constriction of blood vessels.

For these reasons, the effect of TA without epinephrine was assessed by using a fixed composition of the injected solution with the same dilution of anesthetic (ropivacaine 7,5\%; $150 \mathrm{mg}$ ) in $250 \mathrm{cc}$ of saline solution. Moreover, in order to minimize the surgeon-related variability that was reported in previous clinical experiences, mastectomy was performed according to a standardized surgical technique with the only difference regarding the use or not of TA in each of two groups of patients, depending on the surgeon's preference.

Firstly, our finding was that TA significantly reduced operative times. Moreover, postoperative pain was lower in these patients, although not statistically significant. This should not be regarded as a failure of TA-related analgesic effect but as the consequence of the confounding variable due to the prosthesis implantation that may certainly increase postoperative pain. Similarly, the overall volume of lymphatic drainage and the subsequent length of hospitalization were practically similar in the two study groups but this may be related to the variable association of different surgical procedures (mastectomy, axillary dissection, and breast reconstruction). Hence, all these factors should be carefully regarded for the stratification of patients eligible for a randomized clinical trial aimed at defining the impact of TA in patients undergoing alloplastic immediate breast reconstruction after SSM or NSM.

Certainly, the rate of early postoperative complications represents a controversial aspect when using TA, especially with regard to the occurrence of superficial and deep wound infections, hematomas, skin necrosis, and seroma formation that might hamper the outcome of breast reconstruction. For instance, Chun et al. reported a significantly increased risk of flap necrosis when TA was used (odds ratio $=3.93 ; 95 \%$ confidence interval=1.91-8.04: $p<0.001)(8)$. However, an important bias of this study was related to the large variability of the surgical team, such that their findings were not consistent with other reports (21-24). In fact, when multiple logistic regression models were applied to larger cohorts of patients with breast cancer undergoing TA prior to mastectomy and immediate breast reconstruction, TA did not represent an independent risk factor for complication after adjusting for potential perioperative confounders and inherent differences between surgeons (10). Moreover, the development of flap necrosis certainly has a multifactorial pathogenesis including: excessive skin tension, seroma or hematoma formation, infection, and ischemia of skin flaps (24). Notably, the vasoconstrictor effect of epinephrine may contribute to flap ischemia, and our findings suggest that the absence of epinephrine may reduce the risk of skin flap necrosis when immediate breast reconstruction is performed. However, use of a moderate intraoperative tissue expander filling and daily postoperative follow-up are still recommended.

\section{Conclusion}

TA without epinephrine in patients undergoing SSM or NSM with immediate breast reconstruction significantly reduced operative time and local skin damage, thus improving the final cosmetic outcome, with no appreciable side-effects. These findings may well represent the basis for conducting a randomized clinical trial in a well-sized sample of patients with breast cancer with the aim of assessing the outcome related to this form of local anesthesia compared to TA with epinephrine or standard dry mastectomy.

\section{Financial Disclosure}

The Authors have no financial interest in any of the products, devices, or drugs mentioned in this article.

\section{References}

1 Conroy PH and O'Rourke J: Tumescent anaesthesia, Surgeon 11: 210-221, 2013.

2 Klein JA: The tumescent technique. Anesthesia and modified liposuction technique. Dermatol Clin 8: 425-437, 1990.

3 Palmieri B, Baitchev G, Grappolini S, Costa A and Benuzzi G: Delayed nipple-sparing modified subcutaneous mastectomy: rationale and technique. Breast J 11: 173-178, 2005.

4 Carlson GW: Total mastectomy under local anaesthesia: The tumescent technique. Breast J 11: 100-102, 2005.

5 Paige KT, Bostwick J 3rd and Bried JT: TRAM flap breast reconstruction: tumescent technique reduces blood loss and transfusion requirement. Plast Reconstr Surg 113: 1645-1649, 2004.

6 Shoher A, Hekier R and Lucci A Jr.: Mastectomy performed with scissors following tumescent solution injection. J Surg Oncol 83: 191-193, 2003

7 Abbott AM, Miller BT and Tuttle TM: Outcomes after tumescence technique versus electrocautery mastectomy. Ann Surg Oncol 19: 2607-2611, 2012.

8 Chun YS, Verma K, Rosen H, Lipsitz SR, Breuing K, Guo L, Golshan M, Grigorian N and Eriksson E: Use of tumescent mastectomy technique as a risk factor for native breast skin flap necrosis following immediate breast reconstruction. Am J Surg 201: 160-165, 2011.

9 Seth AK, Hirsch EM, Fine NA, Dumanian GA, Mustoe TA, Galiano RD, Hansen NM and Kim JY: Additive risk of tumescent technique in patients undergoing mastectomy with immediate reconstruction. Ann Surg Oncol 18: 3041-3046, 2011. 
10 Khavanin N, Fine NA, Bethke KP, Mlodinow AS, Khan SA, Jeruss JS, Hansen NM and Kim JY: Tumescent technique does not increase the risk of complication following mastectomy with immediate reconstruction. Ann Surg Oncol 21: 384-388, 2014.

11 Klein JA: The tumescent technique for liposuction surgery. Am J Cosmet Surg 4: 263-267, 1987.

12 Klein JA: Tumescent technique for local anesthesia improves safety of large-volume liposuction. Plast Reconstr Surg 92: 1085-1098, 1993.

13 Robertson RD, Bond P, Wallace B, Shewmake K and Cone J: The tumescent technique to significantly reduce blood loss during burn surgery. Burns 27: 835-838, 2001.

14 Cartotto R, Musgrave MA, Beveridge M, Fish J and Gomez M: Minimizing blood loss in burn surgery. J Trauma 49: 1034-1039, 2000.

15 Narita M, Sakano S, Okamoto S, Uemoto S and Yamamoto M: Tumescent local anesthesia in inguinal herniorrhapy with a Prolene hernia system: original technique and results. Am J Surg 198: e27-31, 2009.

16 Memetoglu ME, Kurtcan S, Kalkan A and Ozel D: Combination technique of tumescent anesthesia during endovenous laser therapy of saphenous vein insufficiency. Interact Cardiovasc Thorac Surg 11: 774-778, 2010.

17 Staradub VL and Morrow M: Modified radical mastectomy with knife technique. Arch Surg 137: 105-110, 2002.

18 Samper A and Blanch A: Improved subcutaneous mastectomy with hydrodissection of the subcutaneous space. Plast Reconstr Surg 112: 694-695, 2003.
19 Porter K, O’Connor S, Rimm E and Lopez M: Electrocautery as a factor in seroma formation following mastectomy. Am J Surg 176: 8-11, 1998.

20 Turner EJ, Benson JR and Winters ZE: Techniques in the prevention and management of seromas after breast surgery. Future Oncol 10: 1049-1063, 2014.

21 Miller E, Paull DE, Morrissey K, Cortese A and Nowak E: Scalpel versus electrocautery in modified radical mastectomy. Am Surg 54: 284-286, 1988.

22 Kurtz SB and Frost DB: A comparison of two surgical techniques for performing mastectomy. Eur J Surg Oncol 21: 143-145, 1995.

23 Munhoz AM, Gemperli R and Filassi JR: Comment on: use of tumescent mastectomy technique as a risk factor for native breast skin flap necrosis after immediate breast reconstruction. Am J Surg 204: 549-550, 2012.

24 Myers MB, Brock D and Cohn I Jr.: Prevention of skin slough after radical mastectomy by the use of a vital dye to delineate devascularized skin. Ann Surg 173: 920-924, 1971.

Received November 10, 2016

Revised December 5, 2016

Accepted December 6, 2016 